\title{
Ketogenic diet (KD) therapy in the acute phase of febrile infection-related epilepsy syndrome (FIRES): a case report
}

\author{
Wen-Jing $\mathrm{Li}^{1} \wedge$, Chun-Ling Xue ${ }^{2}$, Yong Zhang ${ }^{1}, \mathrm{Li}-\mathrm{Hui} \mathrm{Wu}^{2}$, Dong-Mei Chen ${ }^{2}$, Feng Chen ${ }^{1}$, Jing Xu ${ }^{1}$, \\ Zhuo $\mathrm{Li}^{2}$, Hong-Jun Miao \\ ${ }^{1}$ Department of Pharmacy, Children's Hospital of Nanjing Medical University, Nanjing, China; ${ }^{2}$ Department of Emergency, Children's Hospital of \\ Nanjing Medical University, Nanjing, China \\ Correspondence to: Zhuo Li; Hong-Jun Miao. Children's Hospital of Nanjing Medical University, 72 Guangzhou Road, Nanjing 210008 , China. \\ Email: lizhuo2004@126.com; jun848@126.com.
}

\begin{abstract}
Management of frequent epileptic seizures in febrile infection-related epilepsy (FIRES) is often challenging. FIRES is an uncommon disease condition. Children with FIRES develop refractory epilepsy with severe cognitive deficits that affect the function of the temporal and frontal lobes. However, better seizure control during the acute stage of FIRES could protect against injury to the nervous system. Ketogenic diet (KD) can effectively resolve super-refractory status epilepticus (SRSE) in the acute phase and improve the prognosis of FIRES. We present the case of a previously healthy 3-year-old male with newonset status epilepticus (SE) admitted to the paediatric intensive care unit for 55 days. Despite treatment with multiple anti-epileptic agents in addition to IV anaesthetics, the patient remained in SRSE and continued to have generalised epileptic activity on electroencephalography (EEG). KD therapy was initiated on the 14th day of the onset, and the patient achieved complete neurological recovery following the KD. Throughout the remainder of admission, the patient was successfully weaned off the ventilator, tolerated oral meals, and worked with occupational and physical therapists to return to his baseline functional status. The convulsions were well controlled after discharge. We discuss the treatment strategies for FIRES and highlight the role of KD therapy in the acute phase to control disease progression and improve the prognosis, and early diagnosis of FIRES and early initiation of KD therapy combined with anti-epileptic drugs (AEDs) could improve the prognosis.
\end{abstract}

Keywords: Febrile infection-related epilepsy syndrome (FIRES); status epilepticus (SE); ketogenic diet (KD); acute phase; case report

Submitted Mar 25, 2021. Accepted for publication Jun 29, 2021.

doi: $10.21037 /$ tp-21-121

View this article at: https://dx.doi.org/10.21037/tp-21-121

\section{Introduction}

Febrile infection-related epilepsy syndrome (FIRES) describes an acute-onset, potentially fatal epileptic encephalopathy that develops in previously healthy children and adolescents during or soon after afebrile episode or any other infectious disease. Children with FIRES develop refractory epilepsy with severe cognitive deficits that affect the function of the temporal and frontal lobes (1). The annual incidence of FIRES among children and adolescents is $1: 1,000,000$ and 1:100,000, respectively (2). FIRES mainly affects children aged 3-15 years old (3), with a peak during school age and male predominance (4).

The proposed consensus definition of 2018 identifies FIRES as a subcategory of new-onset refractory status epilepticus (NORSE) (5), with a history of febrile infection, a history of fever between 2 weeks and $24 \mathrm{~h}$ prior to the

^ ORCID: 0000-0003-1684-4390. 
onset of refractory status epilepticus (SE), and the seizure during the epileptic episode is often refractory to drugs. The pathogenesis underlying FIRES is still a matter of debate, and the clinical outcome of FIRES is poor (2).

The most important treatment for FIRES is the control of epileptic seizures, especially during the acute phase (6). Although general expert consensus in the literature recommends the administration of anti-epileptic drugs (AEDs) or anticonvulsants, they are ineffective in most cases of FIRES (7). However, better seizure control during the acute stage of FIRES could protect against injury to the nervous system. Ketogenic diet (KD) can effectively resolve super-refractory status epilepticus (SRSE) in the acute phase and improve the prognosis of FIRES.

Herein, we report the case of a 3-year-old boy with FIRES, in whom we focused on KD therapy in the acute phase and ultimately achieved complete neurological recovery after extensive treatment.

\section{Case presentation}

\section{Clinical data}

A 3-year-old boy with normal development and no other symptoms of metabolic disease had an onset of intermittent fever for 11 days, which quickly progressed to seizures.

His vital signs included a temperature of $38.5^{\circ} \mathrm{C}$, blood pressure of $112 / 72 \mathrm{mmHg}$, a pulse of 153 beats per minute, and a respiratory rate of 20 breaths per minute. A venous blood gas analysis showed a sodium level of $129 \mathrm{mmol} / \mathrm{L}$, potassium $4.3 \mathrm{mmol} / \mathrm{L}$, and $\mathrm{pH}$ 7.33. The results of the investigations revealed a white blood cell (WBC) count of $14.12 \times 10^{9} / \mathrm{L}$ (reference range $4 \times 10^{9}-10 \times 10^{9} / \mathrm{L}$ ), chest $\mathrm{X}$-ray films revealed dense flocculent shadow in both the lungs, electroencephalography (EEG) revealed a generalised background slowing and frequent right-sided electrical activity, magnetic resonance imaging (MRI) showed clear atrophy, and a lumbar puncture followed by an analysis of the cerebrospinal fluid showed normal results. Based on these, the diagnosis was central nervous system infection, viral encephalitis, SE, and bronchopneumonia.

\section{Treatment}

Anti-infectives, anti-epileptics, and relevant symptomatic treatment were administered.

\section{Anti-infective therapy}

Given the risk of the possibility of co-infection with bacteria, viruses, and mycoplasma, the patient was immediately initiated on anti-infective therapy.

The patient was treated empirically with cefodizime following admission to our pediatric intensive care unit, considering his body temperature of $38.5^{\circ} \mathrm{C}$ and WBC count of $14.12 \times 10^{9} / \mathrm{L}$. However, he continued to have a recurrent fever with a higher thermal peak than before, and cefodizime was changed to meropenem. Due to the drugdrug interactions between meropenem and anti-epileptic agents affecting seizure control, ceftazidime was resumed again when the infection was better controlled following three consecutive routine blood examinations. Ceftazidime was discontinued once the results of the routine blood tests showed a return to normal values, and no abnormalities were found on chest radiography after 2 weeks of treatment. However, his fever recurred 3 days later with a febrile peak of $38.5^{\circ} \mathrm{C}$. The results of routine blood examination showed C-reactive protein (CRP) of $18 \mathrm{mg} / \mathrm{L}, \mathrm{WBC}$ count of $19.65 \times 10^{9} / \mathrm{L}$, and $0.991 \mathrm{ng} / \mathrm{mL}$ of procalcitonin (PCT), and the sputum culture identified Burkholderia cepacia. Based on the results of the drug susceptibility test, it was decided to administer meropenem. After a 5-day treatment, meropenem was changed to ceftazidime when the results of the blood culture were negative for Burkbolderia cepacia but showed sensitivity to ceftazidime. Anti-infective treatment was intensified by the addition of sulfamethoxazole tablets because the tracheal intubation culture was positive for Burkholderia cepacia and Staphylococcus aureus, and the CRP level and WBC counts were higher than before. After treatment with ceftazidime and compound sulfamethoxazole tablets for 3 weeks, the patient was discharged when his health was in better condition.

Drug selection and dose adjustment were tailored according to the clinical manifestations, indicators of infection, and results of the culture. The anti-infective treatment regimen is listed in (Table 1), and the indicators of infections and body temperature during the anti-infective therapy are shown in (Figure 1).

The patient was treated with acyclovir and voriconazole for 3 weeks because he tested positive for Epstein-Barr (EB) virus and a fungus, and erythromycin for 2 weeks because he tested positive for Mycoplasma pneumoniae IgG.

\section{Anti-epileptic therapy}

Despite broad-spectrum AED therapies (topiramate, levetiracetam, phenytoin sodium, and clonazepam), the patient had frequent epileptic seizures, especially tonicclonic seizures. A midazolam infusion was initiated and up- 
Table 1 Anti-infective treatment regimen of the patient during hospitalization

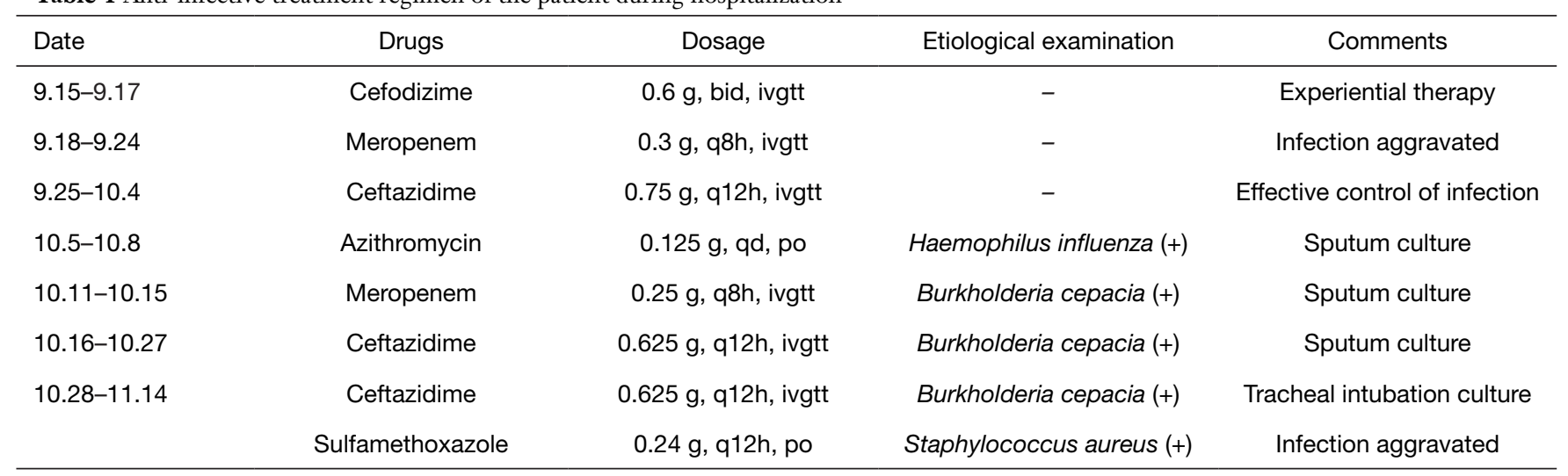
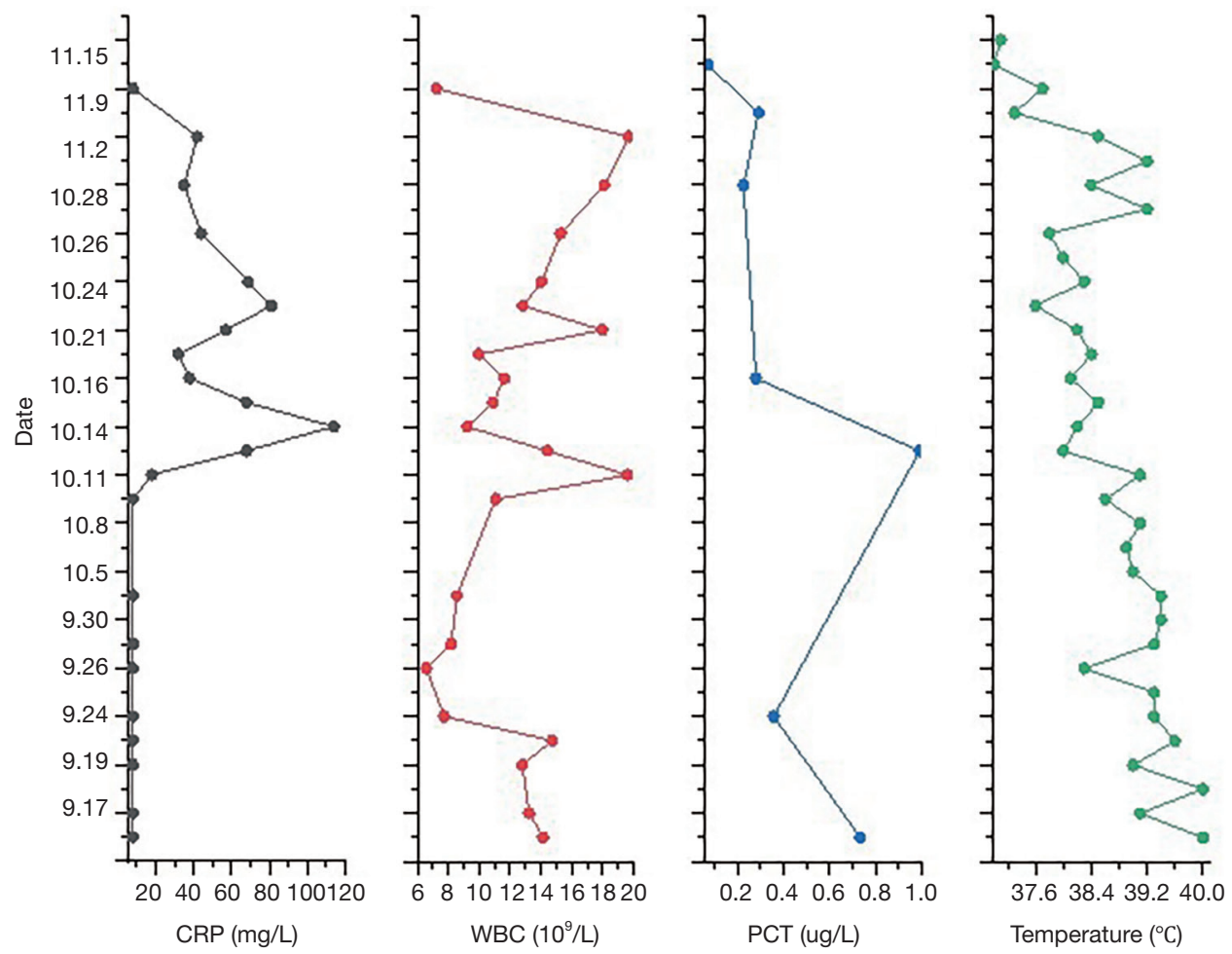

Figure 1 The infectious indicators and body temperature of the patient during hospitalization. CRP, C-reactive protein; WBC, white blood cell; PCT, procalcitonin.

titrated to $0.3 \mathrm{mg} \mathrm{kg}^{-1} \mathrm{~h}^{-1}$; however, it failed to achieve burst suppression, and the patient continued to have generalised epileptic activity on EEG.

While these agents were being titrated, KD therapy was initiated on the 14th day of the onset, and screening for hematuria and B-scan examination of the hepatobiliary tree, pancreas, and spleen were performed before initiating $\mathrm{KD}$ therapy.

The classic KD calculates the ratio of grams of fat to grams of carbohydrates plus protein. The most feasible ratios calculated to date are $3: 1$ or $4: 1$, with approximately $80 \%-90 \%$ of the energy provided by fats and $10 \%$ by carbohydrates and proteins collectively (8). The patient received enteral $\mathrm{KD}$ (EN KD) consisting of a commercially 
Table 2 The formula of KD therapy and the improving symptom of seizure

\begin{tabular}{|c|c|c|c|c|c|c|c|}
\hline Date & $\begin{array}{l}\text { Seizure } \\
\text { frequency }\end{array}$ & $\begin{array}{l}\text { Seizure } \\
\text { types }\end{array}$ & $\begin{array}{c}\text { Grand } \\
\text { mal }\end{array}$ & $\begin{array}{l}\text { Relief time } \\
\text { of seizure }\end{array}$ & \multicolumn{3}{|c|}{ Formula of KD (q3h) } \\
\hline $9.15-9.27$ & Continuous & a & $\sqrt{ }$ & $1-5 \min$ & - & - & - \\
\hline 9.28 & Continuous & a & $\sqrt{ }$ & $1-5 \min$ & 6.8 & 0.5 & 50 \\
\hline 9.29 & Continuous & a & $\sqrt{ }$ & $1-5 \min$ & 13.5 & 1.0 & 70 \\
\hline $10.5-10.7$ & Continuous & $b$ & - & $1-3 \min$ & 14.0 & 0.4 & 70 \\
\hline $10.8-10.11$ & Paroxysmal & c & - & $1-2 \min$ & 14.0 & 0.4 & 70 \\
\hline $10.12-10.17$ & Paroxysmal & c & - & $1-2 \min$ & 15.5 & - & 80 \\
\hline $10.18-10.20$ & Paroxysmal & c & - & $1-2 \min$ & 16.5 & - & 80 \\
\hline 10.28 & Occasional & $d$ & - & $30 \mathrm{~s}$ & 11.0 & 11.0 & 90 \\
\hline $10.29-10.30$ & Occasional & $d$ & - & $15 \mathrm{~s}$ & - & 22.0 & 90 \\
\hline $10.31-11.5$ & Occasional & e & - & $15 s$ & - & - & - \\
\hline $11.6-11.14$ & - & - & - & - & - & - & - \\
\hline
\end{tabular}

$a$, visible generalised convulsions, elevated muscle tone, and dribbling at the mouth; $b$, rhythmic muscle twitches, elevated muscle tone, and tonic eye deviation; c, rhythmic muscle twitches, twitching in the face and nystagmoid, or rhythmic eye movements; $d$, Elevated muscle tone, gazed in both eyes and skewed angles of the mouth; e, rhythmic muscle twitches and dribbling at the mouth. KD, ketogenic diet.

available ketogenic formula (KD milk powder) and a commercially available fat emulsion with medium-chain triglycerides (Peptamen Junior). His body temperature was stable, and the seizure frequency, seizure type, and intervals between the seizures were significantly improved after approximately 1 -month of KD therapy. The formula for KD therapy and the corresponding outcome are listed in (Table 2).

During the KD therapy, electrolytes, arterial blood gases, serum ketone bodies, and glucose were monitored, and these indicators were checked again once the blood ketone level was above $5.5 \mathrm{mmol} / \mathrm{L}$ or the blood glucose level was below $2.5 \mathrm{mmol} / \mathrm{L}$. There were no symptoms of hypoglycaemia or hyper ketoacidosis with blood ketone level of $1.2-4.9 \mathrm{mmol} / \mathrm{L}$ and blood glucose $2.7-7.9 \mathrm{mmol} / \mathrm{L}$.

\section{Relevant symptomatic treatment}

The patient was administered mannitol and glycerol fructose alternately to reduce the intracranial pressure, and the dosage and frequency were gradually reduced according to the clinical status during the treatment. The combination of dextro-ibuprofen suppository with physical cooling was effective in reducing the body temperature with recurrent fever during the course of the disease.

\section{Prognosis}

Throughout the remainder of admission, the patient was successfully weaned off the ventilator, tolerated oral meals, and worked with occupational and physical therapists to return to his baseline functional status. He was continued on topiramate, levetiracetam, phenytoin sodium, and clonazepam and was ultimately discharged on the AED regimen.

The patient continued rehabilitation after discharge from the hospital. Follow-up visits were made 1 to 2 months after discharge and the convulsions were well controlled; however, the patient was lost to follow-up 6 months after discharge.

\section{Discussion}

We present a case of a rare epilepsy syndrome with unclear aetiology in a healthy boy who ultimately recovered completely after extensive treatment, especially $\mathrm{KD}$ 
therapy, during the acute phase. The clinical outcome warrants discussion as FIRES is typically considered a life-threatening condition with mortality up to $30 \%$ and intellectual disability in $66-100 \%$ of the survivors $(9,10)$.

Since there are no known causes, and no disease markers or genetic testing are available for the diagnosis, FIRES is typically diagnosed at a late stage in the clinical course (4), which is primarily based on the clinical status in children with preceding fever who develop refractory SE after known causes of SE have been excluded (9).

In this particular case of FIRES, none of the antiepileptic therapeutic approaches appeared to be significantly effective, with the exception of KD.

$\mathrm{KD}$ contains high fat content, low carbohydrate content, and adequate protein content. It alters the primary cerebral energy supply from glucose to ketone bodies, mimicking biochemical changes during starvation $(11,12) . \mathrm{KD}$ is the most effective when taken after fasting or when the body's calorie levels are low. This is because the brain normally uses glucose as a source of energy rather than fats. However, glucose levels are low in the fasting state; thus, the brain utilises fats as the source of energy in such circumstances (13). Although the exact mechanisms underlying the anticonvulsive activity of $\mathrm{KD}$ are still unclear, it is thought that $\mathrm{KD}$ has anti-epileptic activity, anti-inflammatory effects, and neuroprotective activity (14-17), making it a nonpharmacological substitute for the treatment of epilepsy. Several recent studies have indicated that KD is not only suitable for acute phase therapy but also long-term disease management $(18,19)$. In addition to traditional AEDs, early KD treatment was helpful for both seizure control and cognitive outcome after FIRES $(20,21)$.

\section{Conclusions}

In summary, this challenging case of FIRES in a previously healthy boy emphasises the importance of early initiation of KD in the acute phase. Despite the high risk of mortality and the difficulty in controlling frequent epileptic seizures, clinical outcome resulted in complete neurological recovery. This case also showed that KD was an effective therapeutic option for FIRES, and early diagnosis of FIRES and early initiation of KD therapy combined with AEDs could improve the prognosis.

\section{Acknowledgments}

Funding: None.

\section{Footnote}

Reporting Checklist: The authors have completed the CARE reporting checklist. Available at https://dx.doi. org/10.21037/tp-21-121

Peer Review File: Available at https://dx.doi.org/10.21037/ tp-21-121

Conflicts of Interest: All authors have completed the ICMJE uniform disclosure form (available at https://dx.doi. org/10.21037/tp-21-121). The authors have no conflicts of interest to declare.

Ethical Statement: The authors are accountable for all aspects of the work in ensuring that questions related to the accuracy or integrity of any part of the work are appropriately investigated and resolved. All procedures performed in studies involving human participants were in accordance with the ethical standards of the institutional and/or national research committee(s) and with the Helsinki Declaration (as revised in 2013). Written informed consent was obtained from the patient.

Open Access Statement: This is an Open Access article distributed in accordance with the Creative Commons Attribution-NonCommercial-NoDerivs 4.0 International License (CC BY-NC-ND 4.0), which permits the noncommercial replication and distribution of the article with the strict proviso that no changes or edits are made and the original work is properly cited (including links to both the formal publication through the relevant DOI and the license). See: https://creativecommons.org/licenses/by-nc-nd/4.0/.

\section{References}

1. Kramer U, Chi CS, Lin KL, et al. Febrile infection-related epilepsy syndrome (FIRES): pathogenesis, treatment, and outcome: a multicenter study on 77 children. Epilepsia 2011;52:1956-65.

2. van Baalen A, Häusler M, Plecko-Startinig B, et al. Febrile infection-related epilepsy syndrome without detectable autoantibodies and response to immunotherapy: a case series and discussion of epileptogenesis in FIRES. Neuropediatrics 2012;43:209-16.

3. van Baalen A, Vezzani A, Häusler M, et al. Febrile Infection-Related Epilepsy Syndrome: Clinical Review and Hypotheses of Epileptogenesis. Neuropediatrics 
2017;48:5-18.

4. Gaspard N, Hirsch LJ, Sculier C, et al. New-onset refractory status epilepticus (NORSE) and febrile infection-related epilepsy syndrome (FIRES): State of the art and perspectives. Epilepsia 2018;59:745-52.

5. Hirsch LJ, Gaspard N, van Baalen A, et al. Proposed consensus definitions for new-onset refractory status epilepticus (NORSE), febrile infection-related epilepsy syndrome (FIRES), and related conditions. Epilepsia 2018;59:739-44.

6. Serino D, Santarone ME, Caputo D, et al. Febrile infection-related epilepsy syndrome (FIRES): prevalence, impact and management strategies. Neuropsychiatr Dis Treat 2019;15:1897-903.

7. Sculier C, Gaspard N. New onset refractory status epilepticus (NORSE). Seizure 2019;68:72-8.

8. Starnes K, Miller K, Wong-Kisiel L, et al. A Review of Neurostimulation for Epilepsy in Pediatrics. Brain Sci 2019;9:283.

9. Caraballo RH, Reyes G, Avaria MF, et al. Febrile infection-related epilepsy syndrome: a study of 12 patients. Seizure 2013;22:553-9.

10. Appenzeller S, Helbig I, Stephani U, et al. Febrile infection-related epilepsy syndrome (FIRES) is not caused by SCN1A, POLG, PCDH19 mutations or rare copy number variations. Dev Med Child Neurol 2012;54:1144-8.

11. Klepper J. Glucose transporter deficiency syndrome (GLUT1DS) and the ketogenic diet. Epilepsia 2008;49 Suppl 8:46-9.

12. Yang H, Wu J, Guo R, et al. Glycolysis in energy

Cite this article as: $\mathrm{Li} \mathrm{WJ}$, Xue CL, Zhang Y, Wu LH, Chen DM, Chen F, Xu J, Li Z, Miao HJ. Ketogenic diet (KD) therapy in the acute phase of febrile infection-related epilepsy syndrome (FIRES): a case report. Transl Pediatr 2021;10(9):2392-2397. doi: $10.21037 /$ tp-21-121 metabolism during seizures. Neural Regen Res 2013;8:1316-26.

13. Anwar H, Khan QU, Nadeem N, et al. Epileptic seizures. Discoveries (Craiova) 2020;8:e110.

14. Liu F, Peng J, Zhu C, et al. Efficacy of the ketogenic diet in Chinese children with Dravet syndrome: A focus on neuropsychological development. Epilepsy Behav 2019;92:98-102.

15. Nabbout R, Mazzuca M, Hubert P, et al. Efficacy of ketogenic diet in severe refractory status epilepticus initiating fever induced refractory epileptic encephalopathy in school age children (FIRES). Epilepsia 2010;51:2033-7.

16. Chang P, Augustin K, Boddum K, et al. Seizure control by decanoic acid through direct AMPA receptor inhibition. Brain 2016;139:431-43.

17. Dupuis N, Curatolo N, Benoist JF, et al. Ketogenic diet exhibits anti-inflammatory properties. Epilepsia 2015;56:e95-8.

18. Singh RK, Joshi SM, Potter DM, et al. Cognitive outcomes in febrile infection-related epilepsy syndrome treated with the ketogenic diet. Pediatrics 2014;134:e1431-5.

19. Nangia S, Caraballo RH, Kang HC, et al. Is the ketogenic diet effective in specific epilepsy syndromes? Epilepsy Res 2012;100:252-7.

20. Fox K, Wells ME, Tennison M, et al. Febrile InfectionRelated Epilepsy Syndrome (FIRES): A Literature Review and Case Study. Neurodiagn J 2017;57:224-33.

21. Appavu B, Vanatta L, Condie J, et al. Ketogenic diet treatment for pediatric super-refractory status epilepticus. Seizure 2016;41:62-5. 